\title{
UPDATING OF THE ANATOMY OF THE EXTENSOR MECHANISM OF THE KNEE USING A THREE-DIMENSIONAL VIEWING TECHNIQUE
}

Diego Costa Astur', Saulo Gomes Oliveira², Ricardo Badra², Gustavo Gonçalves Arliani', Camila Cohen Kaleka ${ }^{3}$, Wahi Jalikjian ${ }^{4}$, Pau Golanó ${ }^{5}$ Moisés Cohen 6

\section{ABSTRACT}

The knee extensor mechanism is a complex structure formed by the quadriceps muscle and tendon, the patella, the patellar tendon and the ligaments that surround and help stabilize the knee. Through using a three-dimensional viewing technique on images of the knee extensor apparatus, we aimed to didactically show the structures that compose this bone-muscle-ligament complex. Anatomical dissection of the knee with emphasis on the structures of its extensor mechanism was performed, followed by taking photographs using a camera and lenses suitable for simulating human vision, through a technique for constructing three-dimensional images. Then, with the aid of appropriate software, pairs of images of the same structure from different angles simulating human vision were overlain with the addition of polarizing layer, thereby completing the construction of an anaglyphic image. The main structures of the knee extensor mechanism could be observed with a three-dimensional effect. Among the main benefits relating to this technique, we can highlight that in addition to teaching and studying musculoskeletal anatomy, it has potential use in training for surgical procedures and production of images for diagnostic tests.

Keywords - Knee; Imaging, Three-Dimensional; Anatomy

\section{INTRODUCTION}

The extensor mechanism of the knee is a complex structure formed basically by three structures that are interlinked with each other: quadriceps muscle and tendon, patella and patellar tendon. In addition to these, the mechanism also includes the patellar retinacula, restrictor ligaments, Hoffa's fat pad and the pre-patellar tissue ${ }^{(1,2)}$.
The extensor apparatus is responsible for knee extension and stabilization of the patellofemoral joint. Loss of joint congruence and stability failure between the patella and femur are abnormalities resulting from lack of synergy between the structures of this mechanism. These abnormalities may be congenital or may be due to traumatic episodes of patellofemoral dislocation. Because of the high

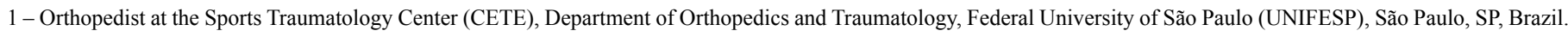

2 - Orthopedist at the Cohen Institute, São Paulo, SP, Brazil.

3 - Orthopedist at the School of Medical Sciences, Santa Casa de Misericórdia de São Paulo, São Paulo, SP, Brazil.

4 - Orthopedist at the School of Medicine of São José do Rio Preto, Ribeirão Preto, SP, Brazil.

5 - Anatomist at the University of Barcelona, Spain.

6 - Full Professor in the Department of Orthopedics and Traumatology, and Head of the Sports Traumatology Department (CETE), UNIFESP, São Paulo, SP, Brazil.

Work performed at the Federal University of São Paulo (UNIFESP/EPM) and at the University of Barcelona.

Correspondence: Rua Borges Lagoa 783, $5^{\circ}$ andar, Vila Clementino, 04038-032 São Paulo, SP. E-mail: mcastur@yahoo.com

Work received for publication: June 22, 2011; accepted for publication: August 25, 2011.
}

The authors declare that there was no conflict of interest in conducting this work 
incidence of patellofemoral disorders, there is great interest in understanding the anatomy of the structures that make up the extensor mechanism and the function of each of these structures ${ }^{(3)}$.

Despite several classical anatomical studies on cadavers, doubts persist regarding the presence, location, anatomical variations and function of some structures in the joint dynamics of the knee extensor mechanism ${ }^{(2,4-6)}$.

The aim of our study was to demonstrate the anatomy of the extensor apparatus by means of three-dimensional photographs, highlighting the medial and lateral ligament structures between the patella, tibia and femur and the vascular pattern responsible for irrigation of this important and complex musculoskeletal grouping, thereby making it easier to understood and study the three-dimensional locations of these structures.

\section{METHODS}

This research project was evaluated and approved by the Ethics Committee of the Federal University of São Paulo, under the number 1801-1810 CEP.

We selected 10 knees from adult cadavers aged 34 to 55 years (mean: 41.2 years), without any history of previous injuries or systemic diseases. The skin and subcutaneous tissue were removed, while preserving the muscle groups and the ligament parts with their neurovascular ramifications.

All the arteries and arterioles were ligated, except for the proximal extremity of the popliteal artery, which was used for insertion of contrast medium composed of transparent liquid silicone, biological catalyst and red or black stain, in order to study the vascularization of the extensor mechanism.

Specific images of the different layers and structures of the anterior compartment of the knee were obtained using a Nikon D40 camera with an AF-S Nikkor 18-55 mm lens (1:3.5 - 5.6 GII ED) and a Micro Nikkor 105 mm lens(1:2.8) (Nikon Corp., Japan).

The camera, with its respective lenses, was attached to a sliding bar that was held on a photographic tripod. The sliding bar was used to furnish two images of the same structure from different angles but at the same height and distance from the structure under examination. The different images on the same structure needed to be separated from each other at distances proportional to the interpupillary distance, with the same convergence as in human vision (62 to $66 \mathrm{~mm}$ ). The pairs of images were processed with the aid of special software named Callipyan 3D or AnaBuilder (AnaBuilder, France). This software is capable of generating a polarizing layer of different colors for each of the images (red for vision from the left eye and blue for vision from the right eye, for example). Following this, superimposition of the polarized images generates a three-dimensional effect when viewed with the aid of appropriate three-dimensional spectacles.

\section{RESULTS}

Three-dimensional images of the main structures of the extensor apparatus of the knee using the technique described above. Figures 1, 2a and $2 b$ show the frontal and medial aspects of the vascularization of the knee extensor mechanism. The medial patellofemoral ligament and medial patellotibial ligament, which are medial restraints for the extensor mechanism, can be seen in Figures 3 and 4. The lateral patellofemoral ligament, which is a lateral restraint for the extensor mechanism, can be seen in Figure 5. The descending genicular artery is highlighted in Figure 6.

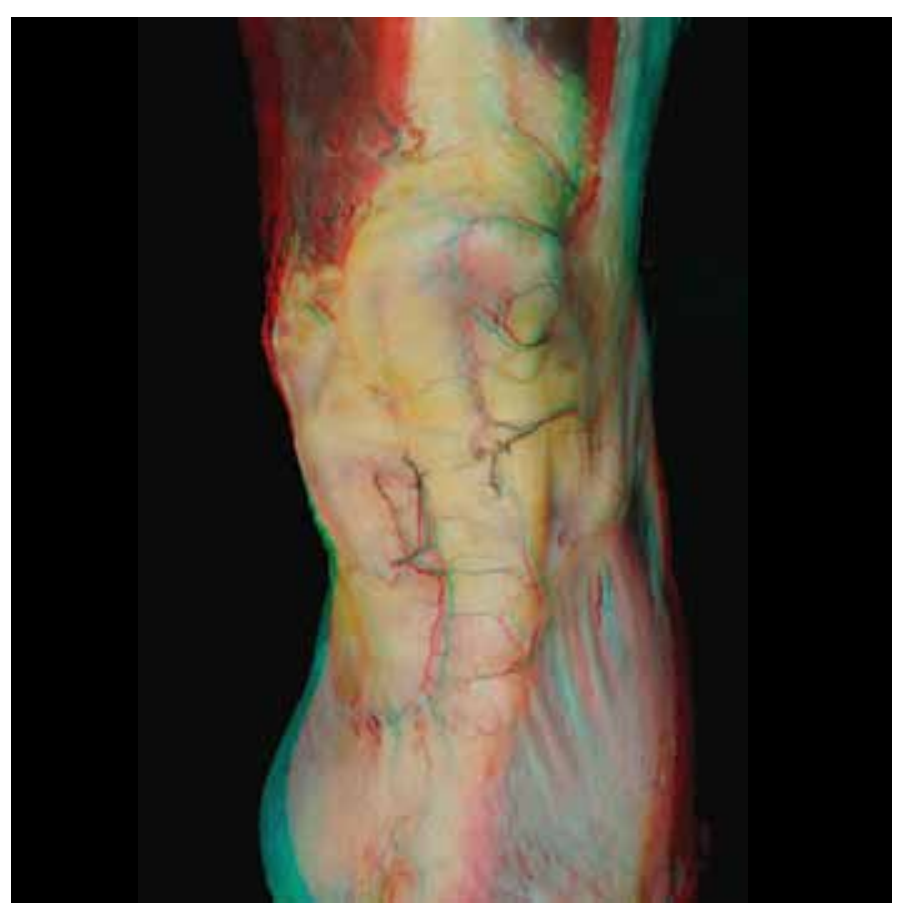

Figure 1 - Frontal appearance of the vascularization of the knee extensor mechanism. 


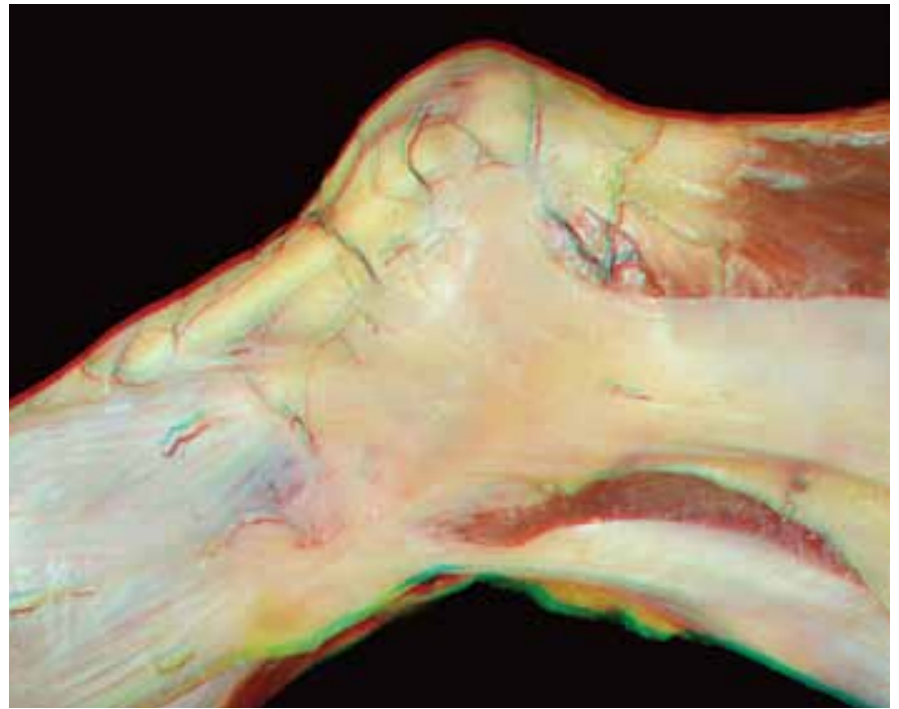

Figure $2 \mathbf{a}$ - Medial view of the vascularization of the knee extensor mechanism: first layer.

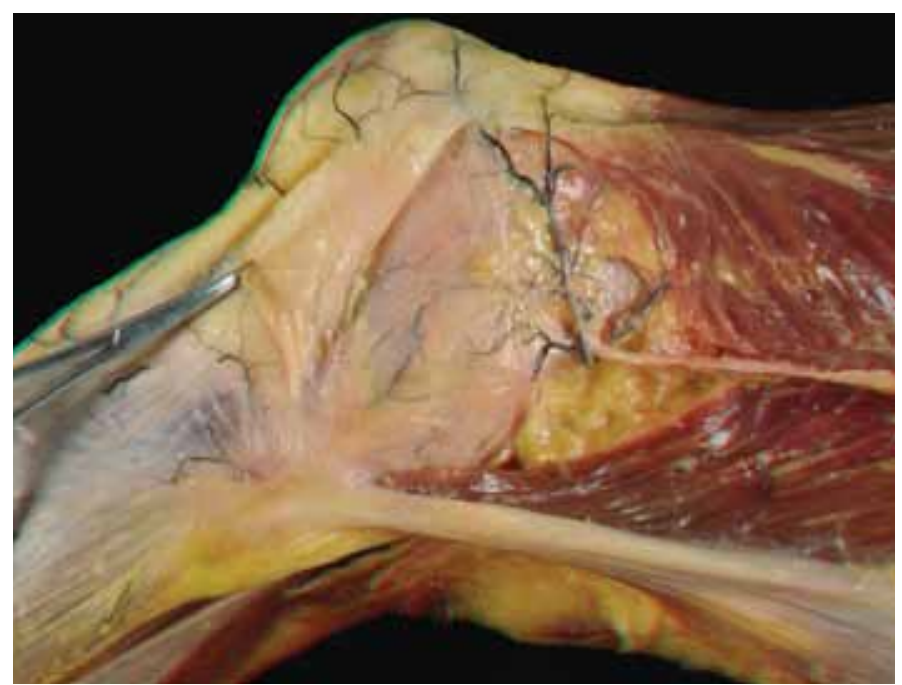

Figure $\mathbf{2 b}$ - Medial view of the vascularization of the knee extensor mechanism: second layer.

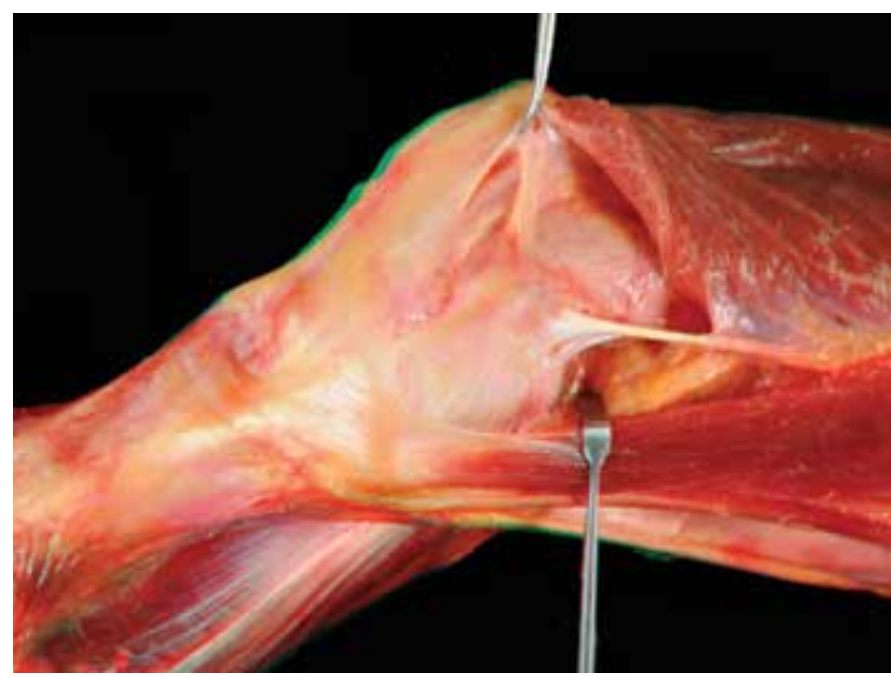

Figure 3 - Medial patellofemoral ligament.

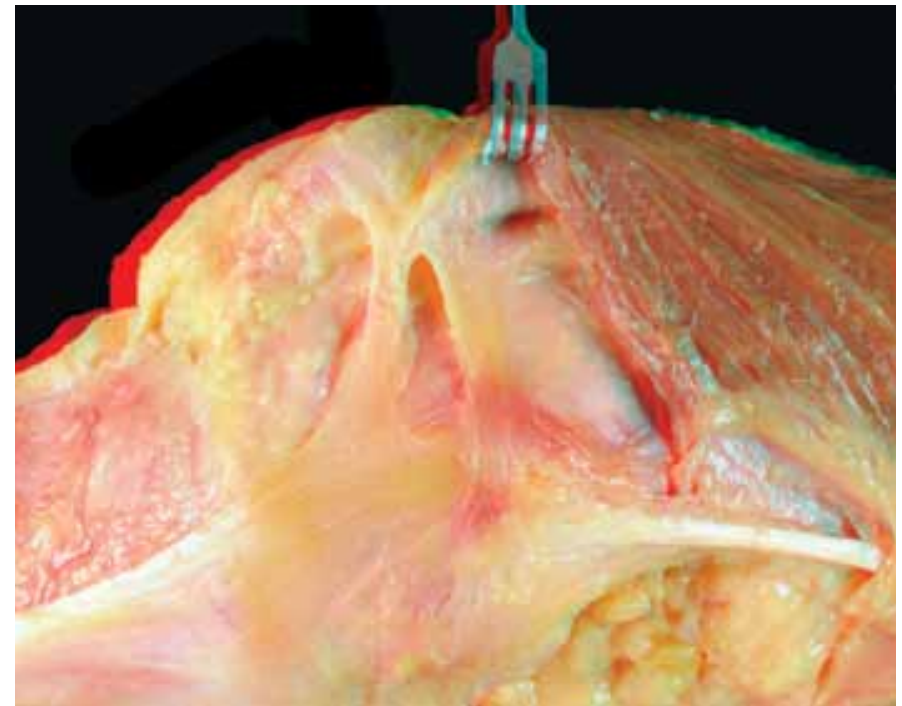

Figure 4 - Medial patellofemoral ligament and medial patellotibial ligament.

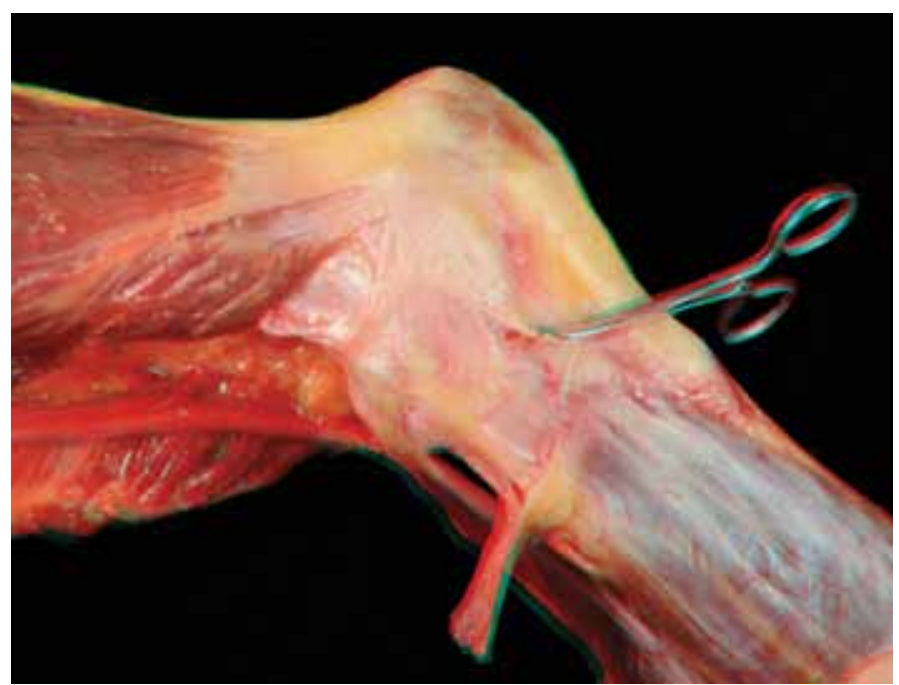

Figure 5 - Lateral patellofemoral ligament.

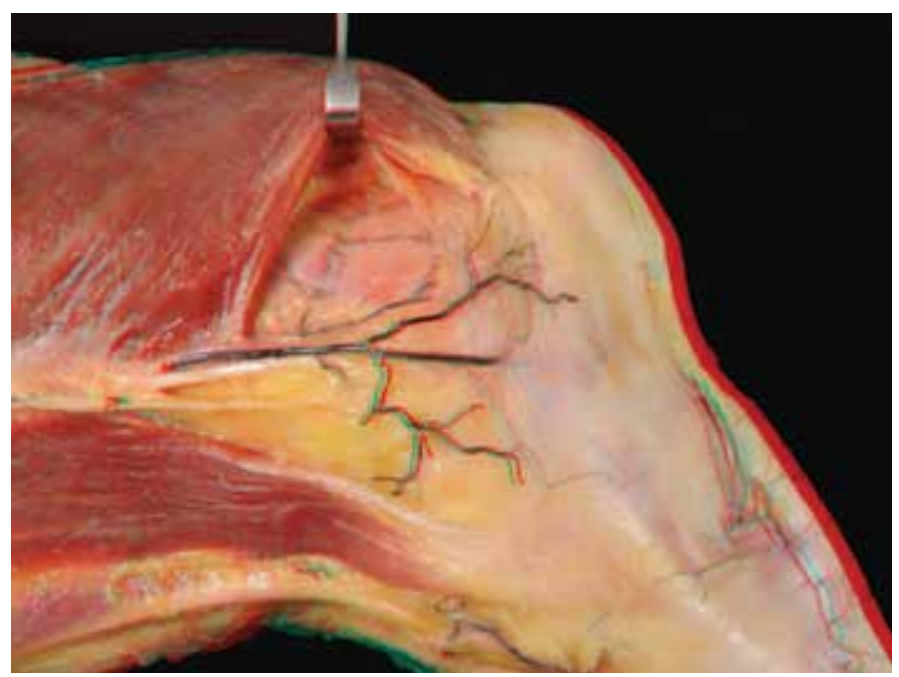

Figure 6 - Descending genicular artery. 


\section{DISCUSSION}

Three-dimensional images enable real discernment of depth, and this is called stereoscopic vision. This type of vision enables orientation of greater precision and interaction with the environment.

Over the last few years, there have been major advances in $3 \mathrm{D}$ image acquisition and refinement, within the fields of entertainment, documentation and teaching. In the medical literature, few studies exploring this technique have been published ${ }^{(7,8)}$, and in the orthopedic literature only a single article has described the technical aspects of acquiring such images $^{(9)}$.

By analyzing these images with the aid of special spectacles, certain concepts in understanding the musculoskeletal anatomy of the knee extensor mechanism can be consolidated.

Anatomically, the quadriceps muscle is basically composed of four muscles. These are the rectus femoris (RF), vastus lateralis (VL), vastus medialis (VM) and vastus intermedius (VI). The fibers of the VL are inserted into the patella at an average of approximately 26 degrees (range: 20 to 38 degree) and the fibers of the VM at 45 degrees (range: 30 to 57 degrees), with the RF and VI as references. These last two are inserted almost perpendicularly into the upper pole of the patella ${ }^{(1,4)}$. Furthermore, the articularis genu muscle (or bursa suspender) originates on the anterior face of the femur. The function of this muscle is to avoid invagination of the suprapatellar bursa under the patella ${ }^{(10)}$.

The more distal and peripheral fibers of the VM and VL muscles presented a direction differing from that of the other central fibers at the insertion on the superomedial and superolateral faces of the patella, respectively, and these are called the vastus medialis obliquus (VMO) and vastus lateralis obliquus (VLO), respectively. Although several anatomical studies describe the existence of these muscles, there is no evidence that these structures are independent from a functional point of view. Rather, they only represent a change in the direction of the more distal fibers before they are inserted into the patella. This arrangement in which some fibers are more oblique contributes towards the dynamic stability of the patella during knee extension $^{(1)}$.

The quadriceps tendon has been described as a trilaminar structure in which the superficial layer is formed by the tendons of the RF muscle, the intermediate layer by the tendons of the VL and VM, and the deep layer by the VI(1). A recent study demonstrated that this classical trilaminar description is in reality a complex structure with large anatomical variation and poorly defined fascial planes ${ }^{(2)}$.

The medial patellofemoral is the main medial restraint on the patella and is responsible for $53 \%$ of the containment force ${ }^{(5)}$. The insertions are located in the medial epicondyle and in the proximal $2 / 3$ of the medial face of the patella $a^{(1,2,5)}$. Other secondary medial stabilizers of the patella that have been described include the medial retinaculum, patellomeniscal ligament and patellotibial ligament ${ }^{(1,5)}$. The medial and the lateral retinaculum are formed by fibers both from the VM, which are parallel to the medial face of the patella, and from the VL, which cross the anterior region of the patella (pre-patella) and are inserted in the contralateral tibia ${ }^{(1,6,11)}$. The medial patellomeniscal ligament originates in the distal third of the patella and is inserted in the anterior cornu of the medial meniscus. The medial patellotibial ligament has been described as originating in the distal and medial regions of the patella, with insertion $1.5 \mathrm{~cm}$ distally to the joint line in the anteromedial region of the tibia ${ }^{(5)}$.

Contrary to the medial compartment, the lateral patellofemoral ligament is not the main restraint on medical displacement of the patella. The resistance that it presents is lower than that of the lateral retinaculum, which is composed of transverse fibers connecting the iliotibial tract to the patella. Lastly, the patellomeniscal ligament is the structure that is least resistant to medial subluxation of the patella ${ }^{(12)}$.

In the pre-patellar region, Dye et $a l^{(6)}$ reported from dissecting cadavers that tissue of trilaminar structure was present. This had a surface layer with transversal fibers, a middle layer with oblique fibers and a deep layer consisting of longitudinal fibers belonging to the RF. Between these layers, three pre-patellar bursas were also present.

The vascularization of the knee and the extensor mechanism is extensive and includes a network of interlinked anastomoses. The arterial supply comes mainly from the femoral, popliteal and anterior tibial arteries. The superficial femoral artery issues a branch named the descending genicular artery, before it crosses the hiatus of the adductors. This artery divides into three branches: saphenous, articular and deep oblique. 
The saphenous branch anastomoses with the medial inferior genicular artery, the articular branch anastomoses with the lateral superior genicular artery and the oblique branch divides into deep muscle branches to feed the quadriceps muscle. The lateral superior genicular, medial superior genicular, lateral inferior genicular and medial inferior genicular arteries, which are branches of the popliteal artery, contribute towards vascularization of the extensor apparatus ${ }^{(13)}$.

The lateral superior genicular artery anastomoses with the descending branch of the lateral femoral circumflex artery, thereby forming, together with other anastomoses, the anastomotic vascular ring of the patella that was described by Scapinelli ${ }^{(14)}$.

The medial and lateral inferior genicular arteries issue branches to feed the patella, Hoffa's fat pad and the patellar tendon. These two arteries each divide into three branches: ascending parapatellar arteries, oblique prepatellar arteries and transverse infrapatellar arteries ${ }^{(13)}$.

The ascending parapatellar arteries follow the edge of the patella, parallel to it, to anastomose with the descending parapatellar branches of the superior genicular arteries. The oblique pre-patellar arteries converge to the anterior region and, together with other vessels coming from other genicular arteries, form the anastomotic vascular ring. Here, on average, 10 to 12 arterial branches penetrate the anterior surface of patella through the vascular foramina. The transverse infrapatellar arteries continue posteriorly to the patellar tendon and originate polar branches that penetrate the cap through the lower

\section{REFERENCES}

1. Andrikoula S, Tokis A, Vasiliadis HS, Georgoulis A. The extensor mechanism of the knee joint: an anatomical study. Knee Surg Sports Traumatol Arthrosc. 2006;14(3):214-20.

2. Waligora AC, Johanson NA, Hirsch BE. Clinical anatomy of the quadríceps femoris and extensor apparatus of the knee. Clin Orthop Relat Res. 2009;467(12):3297-306.

3. Collado H, Fredericson M. Patellofemoral pain syndrome. Clin Sports Med. 2010;29(3):379-98.

4. Reider B, Marshall JL, Koslin B, Ring B, Girgis FG. The anterior aspect of the knee joint. J Bone Joint Surg Am. 1981;63(3):351-6.

5. Conlan T, Garth WP Jr, Lemons JE. Evaluation of the medial soft-tissue restraints of the extensor mechanism of the knee. J Bone Joint Surg Am. 1993;75(5):682-93.

6. Dye SF, Campagna-Pinto D, Dye CC, Shifflett S, Eiman T. Soft-tissue anatomy anterior to the human patella. J Bone Joint Surg Am. 2003;85-A(6):1012-7.

7. Ribas GC, Bento RF, Rodrigues AJ Jr. Anaglyphic three-dimensional stereoscopic printing: revival of an old method for anatomical and surgical teaching and reporting. J Neurosurg. 2001;95(6):1057-66.

8. Meneses MS, Cruz AV, Castro IA, Pedrozo AA. [Stereoscopic neuroanatomy: comparative study between anaglyphic and light polarization techniques]. Arq Neuropsiquiatr. 2002;60(3-B):769-74. pole, posteriorly to the origin of the patellar tendon ${ }^{(13,14)}$.

The patellar tendon receives supplies through three pedicles on each side: superior, medial and inferior. The medial pedicles originate in the descending genicular artery and medial genicular artery, while the lateral pedicles originate in the lateral genicular arteries and the recurrent anterior tibial artery ${ }^{(15,16)}$. Two main vascular arches anastomose with these pedicles: the retropatellar and supratubercular arches, thereby forming a pretendon network ${ }^{(15)}$.

The nerve anatomy of the knee extensor apparatus is composed mainly of the infrapatellar branch of the saphenous nerve, which passes posteriorly to the sartorius muscle on the medial face of the knee, to join with the patellar plexus, thereby supplying innervation to the anteromedial capsule, patellar tendon and anteromedial skin of the knee. The joint nerve branches to the quadriceps muscles, which are branches of the femoral nerve, innervate the anterior and anterolateral faces of the knee ${ }^{(1)}$.

\section{FINAL REMARKS}

Studying the anatomy of the knee extensor apparatus using $3 \mathrm{D}$ photographs makers it possible to see the different structures and understand the relationships between them. Among the benefits of this technique other than in relation to teaching and studying musculoskeletal anatomy, we can highlight its potential use in training for surgical procedures and producing images in diagnostic examinations.
9. Cohen M, da Costa Astur D, Kaleka CC, Arliani GG, Cohen C, Jalikjian W, et al. Introducing 3-dimensional stereoscopic imaging to the study of musculoskeletal anatomy. Arthroscopy. 2011;27(4):593-6.

10. Kimura K, Takahashi Y. M. articularis genus. Observations on arrangement and consideration of function. Surg Radiol Anat. 1987;9(3):231-9.

11. Wangwinyuvirat M, Dirim B, Pastore D, Pretterklieber M, Frank A, Haghighi $P$, et al. Prepatellar quadriceps continuation: MRI of cadavers with Gross anatomic and histologic correlation. AJR Am J Roentgenol. 2009 Mar;192(3):W111-6.

12. Merican AM, Sanghavi S, Iranpour F, Amis AA. The structural properties of the lateral retinaculum and capsular complex of the knee. J Biomech. 2009;42(14):2323-9.

13. Colombel M, Mariz Y, Dahhan P, Kénési $C$. Arterial and lymphatic supply of the knee integuments. Surg Radiol Anat. 1998;20(1):35-40..

14. Scapinelli R. Blood supply of the human patella. Its relation to ischaemic necrosis after fracture. J Bone Joint Surg Br. 1967;49(3):563-70.

15. Soldado F, Reina F, Yuguero M, Rodríguez-Baeza A. Clinical anatomy of the arterial supply of the human patellar ligament. Surg Radiol Anat. 2002;24(34):177-82.

16. Pang J, Shen S, Pan WR, Jones IR, Rozen WM, Taylor GI. The arterial supply of the patellar tendon: anatomical study with clinical implications for knee surgery. Clin Anat. 2009;22(3):371-6. 